\title{
THE ROLE OF INDUSTRY STRUCTURE, COSTS, AND ECONOMIC SPILLOVERS IN DETERMINING STATE EMPLOYMENT GROWTH RATES
}

\author{
Mark D. Partridge and Dan S. Rickman*
}

\begin{abstract}
We examine differential state employment growth by appraising the relative effects of traditional cost factors versus knowledge and technology spillovers. One emphasis is the influence that industry composition has on employment growth. Traditional cost factors examined include wage rates, unionization, taxes, and government policies such as unemployment insurance and welfare programs. The results suggest that industry composition does influence growth, working through several different avenues. In addition, taxes and other cost factors also significantly influence employment growth. Conversely, there was less evidence to suggest that knowledge spillovers play a significant role in determining state employment growth.
\end{abstract}

\section{INTRODUCTION}

Traditional analysis of differences in regional economic growth has focussed on variations in urbanization, costs, industry composition, and amenities. Recently, endogenous growth models have proposed other explanations for regions or nations to experience persistent differences in long-run growth rates or long-run income levels. ${ }^{1}$ Given variations in intensities of R\&D and human capital across industries and regions, endogenous growth models suggest that knowledge spillovers, either across firms within an industry or between industries, could occur that would influence relative regional growth rates. Therefore, this paper attempts to sort out the relative contributions to state economic growth of traditional business location factors and of knowledge and industry spillovers suggested by endogenous growth models.

Garcia-Milà and McGuire (1993) ( $G$ \& M) show that the composition of industries within a state influences economic growth. However, they left unanswered the question of why industrial composition has such an influence on employment growth rates. For example, the influence of industry composition could be caused by multiplier effects, where a fast-growing mix of industries induces faster economic growth in other industries. Alternatively, it could be that certain industries generate more knowledge spillovers either between firms in an

\footnotetext{
*Professor, Department of Economics, St. Cloud State University, and Professor, Department of Economics, Oklahoma State University, Stillwater. An earlier version of this paper was presented at the Southern Regional Science Association Meetings in San Antonio, Texas, April, 1995. We thank David Kraybill and session participants for their helpful comments.
} 
industry (e.g., in Silicon Valley) or across industries. Finally, there could be differences in the demand/supply pull of particular industries on a state's economy. Thus, we also extend Garcia-Milà and McGuire (1993) by sorting out the separate channels through which industrial composition can influence state employment growth.

Traditional business location studies emphasize the importance of cost considerations such as differences in wages, taxes, and unionization. (Bartik, 1991 provides a comprehensive survey of this literature.) A typical hypothesis is that greater wage rates chase away business. However, state and local policymakers desire high-wage jobs, since the additional income from these jobs generates more jobs and improves the standard of living. Thus, we try to simultaneously address the trade-off between the negative cost effects from high wages with their positive multiplier effects.

Therefore, this paper extends previous regional economic literature in the following ways. First, we appraise multiplier effects caused by a mix of fastgrowing industries or by a mix of high-paying industries. Second, we analyze whether the industrial composition of a state has an additional influence on employment growth because each sector could have a different propensity to demand or supply inputs within its respective state or region. Third, we weigh the relative roles of factors emphasized in traditional business location studies against factors stressed in new endogenous growth models. Fourth, we utilize a detailed data set that spans the early 1970 s to the early 1990 s. This time period should be sufficient to capture long-run trends. Moreover, the rich data set contains many variables that should be of direct interest to policymakers.

\section{MODEL OF DIFFERENTIAL STATE EMPLOYMENT GROWTH RATES}

The regional economics literature and endogenous growth models suggest numerous profit-related arguments for economic growth to vary across regions (see Crihfield 1989 for a similar framework). Profitability $\left(\pi_{\mathrm{rt}}\right)$ can be formally expressed for a representative firm in region $r$, period $t$

$$
\pi_{r t}=p_{r t}(.) A(.) \mathrm{F}\left(e_{r l} L_{r t, .}\right)-w_{r l} L_{r t}-C\left(\mathbf{G O V T} \boldsymbol{T}_{r, .}\right)
$$

where $p($.$) is the price received by the firm; A($.$) is a technological shift parameter$ or factor-neutral productivity measure; $F$ is the production function that includes labor $(L)$, the efficiency of labor $(e)$, and other non-labor inputs as arguments (in- 
cluding capital); $w$ denotes wages; and $C$ denotes non-wage costs, including government policies (GOVT) such as taxes, and other non-labor input costs.

From Equation (1), we see that higher wage rates, taxes, and costly government policies reduce profitability and the attractiveness of the region to firms. However, offsetting this are factors that increase productivity, shifting the firm's production function and increasing profitability. Thus, the most profitable location for a firm is influenced by both factors that affect productivity or revenues and elements that affect the unit cost of production.

Several authors have suggested that there are indeed differences across regions that shift the aggregate production function (e.g., Barro and Sala-i-Martin 1991; Beeson 1987; Hulten and Schwab 1984; and Malhotra and Garofalo 1988). However, there are many possible explanations for regional differences in productivity.

One possibility is that the total human capital stock of a region may cause variations in regional productivity (e.g., Lucas 1988; and Rauch 1993). Another possibility is that industry composition influences aggregate productivity (Carlino and Voith 1992). For example, a state with a concentration of high-productivity manufacturing industries will have greater productivity than a state with a concentration of low-productivity service industries. Similarly, knowledge spillovers or dynamic externalities in a region could influence aggregate productivity (e.g., Glaeser et al. 1992). Such spillovers could be between firms within an industry or between industries. Knowledge spillovers are closely related to R\&D spillovers and the availability of a well-trained work force, suggesting that states with a greater concentration of knowledge-intensive high-tech firms may grow at a fasterrate.

Agglomeration effects represent another economic spillover (e.g., Henderson 1994). For instance, the geographic concentration of certain industries, such as financial services, may in turn attract expanding industries in search of financing and venture capital. In a related point, agglomeration effects and knowledge spillovers depend on the industry's product market structure. Large monopolistic firms may have more incentive to conduct R\&D because they face little threat of competitors imitating their innovations. Alternatively, smaller competitive firms may experience more market pressures to innovate (Porter 1990).

Therefore, the technological shift parameter can be written as

$$
A\left(t, \mathbf{R E G}_{r}, \mathbf{H}_{r t}, \mathbf{K}_{r t}, \mathrm{IND}_{r t}, \mathrm{SIZE}_{r t}\right)
$$

where $r$ denotes region, $t$ denotes year; REG represents regional effects; $\mathbf{H}$ is the total human capital of the region; $K$ denotes knowledge and R\&D spillovers; IND is the industry mix of the state; and SIZE represents agglomeration effects in the 
region. Generally, factors that increase $A($.$) also increase the level of profitability,$ which in turn causes firms in region $r$ to expand and induces new firms to locate in region $r$.

In addition, regional output depends not only on the physical quantity of labor, but also on the efficiency $(e)$ of the individual workers in the labor force. Clearly, the efficiency of a worker in the labor force is closely associated with the worker's human capital.

By substituting Equation (2) into Equation (1), the region's labor demand can be derived, where generally labor demand and the level of profitability are assumed to be positively related.

$$
L_{r t}^{D_{r}}=\mathbf{g}\left(p_{r t}, \mathbf{R E G} \mathbf{G}_{r}, \mathbf{H}_{r t}, \mathbf{K}_{r t}, \mathrm{IND}_{r t}, \mathrm{SIZE}_{r t,}, e_{r t}, w_{r t}, \mathbf{G O V T}_{r t, t, .}\right)
$$

Labor supply in a region, of course, is directly related to the region's population and is a function of the human capital and demographic characteristics (DEMOG) of the population, the level of wages in the region, and government policies. In addition, population growth and labor supply are strongly influenced by regional amenities such as climate or scenery. The labor supply of region $r$ is depicted in Equation (4).

$$
L_{r t}^{S}=\mathbf{f}\left(\mathbf{R E G}_{r}, \mathbf{H}_{r t}, w_{r t}, \mathbf{G O V T}_{r t}, \mathbf{D E M O G}_{r t, t, .}\right)
$$

In particular, the level of regional attributes such as amenities, wages, and taxes influence the level of labor supply along with influencing the number of new migrants.

Total employment in a region results from the interaction of labor demand and labor supply. Thus, by incorporating the structural labor supply elements of Equation (4) into Equation (3), a quasi-reduced form for the long-run equilibrium level of employment $E^{*}$ can be obtained.

$$
E_{r t}^{*}=\mathbf{g}^{\prime}\left(p_{r l}, \mathbf{R E G}_{r}, \mathbf{H}_{r t}, \mathbf{K}_{r t}, \mathbf{I N D}_{r t}, \mathbf{S I Z E}_{r t}, e_{r t}, \mathbf{G O V T}_{r}, \mathbf{D E M O G}_{r, t}, .\right)
$$

Equation (5), by considering the equilibrium level of employment $E^{*}$, implicitly assumes that all of the regional labor markets are simultaneously in equilibrium. However, it is unlikely that all state economies are continuously in equilibrium. Specifically, it is likely that regional labor markets regularly undergo disequilibrium adjustments to their evolving long-run equilibrium level of employment (e.g., see Greenwood et al. 1991). Likewise, Partridge and Rickman (1995) found evidence that between the early 1970s and early 1990s, many state and regional economies experienced economic cycles that systematically diverged 
from the national economy (e.g., the oil patch states). Analogously, Barro and Sala-i-Martin (1991) and Crihfield and Panggabean (1995) have found relatively slow convergence of per capita income between regions over time. Thus, allowing for disequilibrium in state employment growth rates seems warranted.

The particular disequilibrium model that we implement is a simple stockflow process, where we assume that the factors that influence $E^{*}$ in Equation (5) also influence the disequilibrium adjustment to $E^{*}$ (for more discussion, see Newman and Sullivan 1988). In addition, we allow annual employment growth to be separately influenced by short- to medium-term exogenous demand shocks that impact current employment growth (as well as the current level of employment, which may or may not equal $E^{*}$ ). Examples of demand shocks include the state's possessing a mix of industries that are faring well at the national level and economic spillovers from nearby states. Finally, in equilibrium, we allow state r's growth rate to equal the national employment growth rate.

The proposed model can be more formally expressed as

$$
E G R W_{r t}=\lambda\left[\left(E_{r t}^{*} E_{r t-1}\right) / E_{r t-1}\right]+\text { DEMAND }_{r t}+E G R W_{U t} ; 0<\lambda<1
$$

where $E G R W_{r t}$ is state r's annual employment growth rate (i.e., $\left.\left(\mathrm{E}_{\mathrm{rt}}-\mathrm{E}_{\mathrm{rt}-1}\right) / \mathrm{E}_{\mathrm{rt}-1}\right)$, DEMAND $_{r t}$ is exogenous demand shocks, EGRWUt is the national nonfarm employment growth rate (i.e., $\left.\left(E_{u t}-E_{u t-1}\right) / E_{u t-1}\right)$, and $\lambda$ is the extent of the disequilibrium level of employment as a proportion of employment in the last period that is eliminated each period (i.e., the speed of adjustment). Regarding the first term in Equation (6), if state r's previous level of employment $E_{r t-1}$ is less than $E^{*}{ }_{r}$, state r's employment growth will exceed the national average in time period $t$, all else being equal. Similarly, when $E_{r t-1}$ is greater than $E_{r t}^{*}$, state r's employment growth will trail the national average. Accordingly, factors that increase $E_{r t}^{*}$ will also increase state r's employment growth and vice versa, where the factors that have been found to affect employment in other studies are incorporated in the analysis. ${ }^{2}$

We obtain our annual measure of disequilibrium state employment growth $\left(N E T G R W_{r t}\right)$ by subtracting the national private nonfarm employment growth rate $\left(E G R W_{U_{t}}\right)$ from both sides of Equation (6):

$$
N E T G R W_{r t}=\lambda\left[\left(E_{r t}^{*} E_{r t-1}\right) / E_{r t-1}\right]+\text { DEMAND }_{r t}
$$

Using NETGRW $W_{r}$, which is annual private state employment growth minus U.S. private employment growth, has the advantage of creating a measure of the disequilibrium from national trends, as well as netting out national cyclical patterns. 
By substituting Equation (5) for $E^{*}$ in Equation (6'), Equation (7) can be obtained

$N E T G R W_{n}=\mathrm{h}\left(p_{n}, \mathbf{R E G}_{r}, \mathbf{H}_{r}, \mathbf{K}_{r}, \mathbf{I N D}_{r}, \mathbf{S I Z E}_{n}, e_{r}, \mathbf{G O V T}_{r}\right.$, DEMOG $\left._{n}, t,.\right)+$ DEMAND $_{n}$

where $\lambda$ is subsumed into $h($.$) and all the measures in h$ are scaled to account for the size of the state. ${ }^{3}$ In Equation (7), cross-state differences in the levels of the control variables are hypothesized to cause changes in state r's annual employment growth relative to the nation (NETGRW). For example, if greater unionization increases costs and reduces the profit rate, then employment in states with relatively high levels of unionization should grow less than the national average, ceteris paribus. Similarly, states with above-average rates of taxation will likely experience less than average net migration flows and smaller than average labor force growth rates, as well as lower average profit rates and less firm growth. Finally, another advantage of using the change in employment and the level of most of the independent variables is that it avoids spurious relationships that may exist between the level of employment and the level of the independent variables (i.e., unit root problems that result from regressing an I(1) variable on other I(1) variables).

To test the sensitivity of our results to the measure of employment growth, we try two different annual measures of relative state employment growth (NETGRW) as our dependent variable. The first measure of relative state economic performance is the competitiveness term from shift-share analysis. ${ }^{4}$ The competitiveness growth rate is the difference between the state's actual growth rate and the hypothetical growth rate that would occur if each of the state's industries grew at their respective national growth rates. In other words, the competitiveness term is a measure of how well a state is faring after adjusting for the national performance of its mix of industries. Competitiveness employment growth should be a good measure of a state's economic health because it adjusts for the possibility that a state is doing well (poorly) solely because it has a mix of industries that are performing strongly (weakly) nationally. ${ }^{5}$ The second measure of differential employment growth is the relative state employment growth rate, which is the annual state employment growth rate net of the national employment growth rate. Because the relative employment growth rate is the sum of the industry mix employment growth rate and the competitiveness employment growth rate, this gauge of employment growth implicitly assumes that the industry mix employment growth rate of a state is endogenous (i.e., it is part of the dependent variable). Therefore, we can judge the robustness of our results by examining both measures of relative state employment growth. 


\section{EMPIRICAL MODEL, VARIABLE DEFINITIONS, AND SOURCES}

To empirically implement Equation (7), a linear specification with an error term is assumed:

$$
N E T G R W_{r t}=\beta_{0}+\beta \mathbf{X}+\sigma_{r}+v_{r t}
$$

where $\beta_{0}$ is a constant, $\beta$ is a coefficient vector, $\mathbf{X}$ is a vector of variables outlined below, and $v_{r t}$ is an error term. State and regional fixed effects are accounted for by $\sigma_{r}$ (i.e., the REG term in (7)), which allows for the possibility of persistent cross-state differences in employment growth during our sample period, which may be due to distance from markets, amenity effects, or threshold size effects. Finally, in the empirical analysis, we will examine whether the error term $u_{r t}$ exhibits first-order autocorrelation:

$$
v_{r t}=\rho v_{r t-1}+u_{r t}
$$

where $u_{r t}$ is i.i.d. and $\rho$ is the degree of first-order autocorrelation that is assumed constant across states.

Data for the 48 contiguous states are drawn from the 1972-1991 period, for a total of 960 observations. Variable definitions and sources are given in Table 1 while the means and standard deviations of the independent variables are shown in column (1) of Table 2. Two different private nonfarm employment dependent variables are derived from U.S. Bureau of Labor Statistics data. The first dependent variable is the competitiveness employment growth rate (COMPETE_EMP) and the second is the relative employment growth rate. By definition, the employment-weighted average of each growth rate measure equals zero. The standard deviation for the competitiveness and relative employment terms are 0.021 and 0.022 , suggesting about a 2 percent standard deviation in state employment growth across states and time.

For the competitiveness employment growth equation, we control for industry mix employment growth (INDMIX_EMP), which measures whether the state has a mix of fast- or slow-growing industries at the national level. INDMIX_EMP is the difference between the hypothetical employment growth rate for a state if each of its industries grew at their respective national employment growth rates and the U.S. total employment growth rate. ${ }^{6}$ To capture dynamic effects, we include the lag of INDMIX_EMP (INDMIX_EMP_LAG). Including industry mix tests that illustrate whether exogenous demand shifts resulting from having a mix of fast- or slow-growing industries at the national level 
TABLE 1

Variable Definitions and Sources

\begin{tabular}{ll} 
VARIABLE & DEFINITION AND SOURCE \\
\hline COMPETE_EMP & The state's actual nonfarm private sector employment \\
growth rate minus the sum of the industrial mix employment \\
growth rate (INDMIX) and the U.S. employment growth \\
rate (US): (COMPETE=ACTUAL STATE GROWTH \\
RATE-INDMIX-US). Source: BEA.
\end{tabular}

INDMIX_EMP

INDUSTRY SHARES

CHANGE_FARM

CHANGE_MILITARY

REGION_GROWTH

HIGH-TECH_SHARE

HERFINDAHL

FIRMSIZE

METRO

LN_SMSA_POP

WAGE_MIX

WAGE_COMP

UNION\%

UI_BENEFIT
The difference between the state's employment growth rate if employment in the state's two-digit industries were growing at their respective average national industry employment growth rates and the U.S. employment growth rate for the nonfarm private sector. Source: Bureau of Economic Analysis (BEA).

The one-digit shares of total civilian employment in the state that are accounted for by agriculture; durable manufacturing; nondurable manufacturing; mining; construction; transportation and public utilities; finance, insurance, and real estate; wholesale and retail trade; services. Source: BEA.

Annual change in the farm employment share. Source: BEA.

Change in military spending as a share of gross state product. Source: Trott et al. (1991).

Employment growth in the Census region of the state, net of the state's employment growth contribution. Source: BEA.

The percent of private nonfarm employment in high-tech manufacturing. Manufacturing industries classified as hightech are from the U.S. Department of Commerce classification. Source: 1993 Statistical Abstract of the United States.

The Herfindahl index is the sum of the squares of the percent of employment in each two-digit nonfarm private sector industry. Source: U.S. Bureau of Economic Analysis (BEA).

Average nonfarm private sector establishment size in the state. Source: County Business Patterns.

Annual percent of the state's population that resides in a metropolitan area. Source: U.S. Department of Commerce.

The natural $\log$ of the 1972 population of the state's largest metropolitan area. Source: Statistical Abstract of the United States.

The state-employment-weighted average of corresponding national industry wage rates expressed relative to the average U.S. private wage rate. Source: Bureau of Labor Statistics, BEA, and U.S. Department of Commerce.

The ratio of the actual state wage rate divided by stateemployment-weighted U.S. industry wage rates.

The percent of the civilian labor force that are union members. Source: U.S. Department of Commerce and Hirsch and Macpherson (1993).

The real average weekly unemployment insurance benefit for each state using the CPI as the deflator. Source: U.S. Department of Commerce and U.S. Employment and Training Administration. 
TABLE 1 (Continued)

Variable Definitions and Sources

\begin{tabular}{ll}
\hline VARIABLE & DEFINITION AND SOURCE \\
\hline UI_COVERAGE & $\begin{array}{l}\text { The percent of civilian employment that is covered by un- } \\
\text { employment insurance in each state. Source: U.S. Depart- } \\
\text { ment of Commerce and U.S. Employment and Training } \\
\text { Administration. }\end{array}$
\end{tabular}

WELFARE_\%INCOME

TAX_\%INCOME

FUEL_COST

INTERNATIONAL_MIG

MALE_LABOR_FORCE\%

BLACK_POP\%

MARRIED\%

FEM_MAR_CHILDU6

FEM_NONMAR_CHILDU6

AGE_OVER64\%

AGE_UNDER15\%

HIGHSCHOOL_GRAD

COLLEGE_GRAD
The percent of personal income accounted for by public welfare expenditures. Source: Government Finances.

State and local taxes as a percent of state personal income.

Source: Government Finances and U.S. Bureau of

Economic Analysis.

Index of the state's fuel costs relative to the national average. Source: Treyz et al. (1992).

Annual international migrants as a share of the state's population. Source: Current Population Survey, various years.

The percent of total civilian employment in the state that is accounted for by males. For some of the smaller states, male employment was unavailable for 1972-1975. In these cases, the earliest year where exact data was available was interpolated with data from the 1970 Census. Source: U.S. Department of Labor; BEA; U.S. Bureau of the Census.

The percent of the population in the state that is black. Data was interpolated using 1970,1980, and 1990 as endpoints. Source: U.S. Department of Commerce.

The percent of women over the age of 16 that are married. Data was interpolated using 1970,1980 , and 1990 as endpoints. Source: U.S. Bureau of the Census.

The percent of women over the age of 16 that are married with children under the age of 7 . Data was interpolated using 1970, 1980, and 1990 as end points. Source: U.S. Bureau of the Census.

The percent of women over the age of 16 that are not married with children under the age of 7. Data was interpolated using 1970, 1980, and 1990 as end points. Source: U.S. Bureau of the Census.

The percent of the population that is 65 years old or older. Source: U.S. Bureau of the Census, Current Population Report.

The percent of the population that is 14 years old or younger. Source: U.S. Bureau of the Census, Current Population Report.

The percent of the population over the age of 24 that are high school graduates. Data was interpolated using 1970 , 1980 , and 1990 as end points. Source: U.S. Department of Commerce.

The percent of the population over the age of 24 that are college graduates. Data was interpolated using 1970,1980, and 1990 as end points. Source: U.S. Department of Commerce. 
TABLE 2

State Competitiveness Employment Regression Results ${ }^{\mathrm{a}, \mathrm{b}}$

\begin{tabular}{|c|c|c|c|c|c|c|c|}
\hline VARIABLE & $\begin{array}{c}(1) \\
\text { MEAN }\end{array}$ & $\begin{array}{l}\text { (2) } \\
\text { OLS }\end{array}$ & $\begin{array}{c}\text { (3) } \\
\text { PANEL }\end{array}$ & $\begin{array}{c}\text { (4) } \\
\text { AR1 } \\
\text { PANEL }\end{array}$ & $\begin{array}{c}(5) \\
\text { AR1 } \\
\text { PANEL }\end{array}$ & $\begin{array}{c}(6) \\
\text { AR1 } \\
\text { PANEL }\end{array}$ & $\begin{array}{c}\text { (7) } \\
\text { 2SLS } \\
\text { AR1 }\end{array}$ \\
\hline \multicolumn{8}{|l|}{$\begin{array}{l}\text { DEMAND OR } \\
\text { STRUCTURAL: }\end{array}$} \\
\hline INDMIX_EMP & $\begin{array}{l}-0.002 \\
(0.005)\end{array}$ & $\begin{array}{l}0.873 \\
(6.15)\end{array}$ & $\begin{array}{c}0.65 \\
(6.15)\end{array}$ & $\begin{array}{c}0.79 \\
(7.80)\end{array}$ & & & $\begin{array}{c}0.81 \\
(8.02)\end{array}$ \\
\hline INDMIX_EMP_LAG & $\begin{array}{l}-0.001 \\
(0.006)\end{array}$ & $\begin{array}{r}0.439 \\
(3.58)\end{array}$ & $\begin{array}{c}0.30 \\
(3.03)\end{array}$ & $\begin{array}{c}0.37 \\
(3.72)\end{array}$ & & & $\begin{array}{c}0.37 \\
(3.73)\end{array}$ \\
\hline FARM_SHARE & $\begin{array}{c}0.016 \\
(0.012)\end{array}$ & $\begin{array}{r}0.143 \\
(1.43)\end{array}$ & $\begin{array}{l}-0.053 \\
(0.30)\end{array}$ & $\begin{array}{l}-0.028 \\
(0.14)\end{array}$ & $\begin{array}{l}-0.064 \\
(0.32)\end{array}$ & & $\begin{array}{l}-0.009 \\
(0.04)\end{array}$ \\
\hline DUR_SHARE & $\begin{array}{c}0.110 \\
(0.050)\end{array}$ & $\begin{array}{r}0.174 \\
(3.56)\end{array}$ & $\begin{array}{r}0.202 \\
(1.95)\end{array}$ & $\begin{array}{r}0.069 \\
(0.68)\end{array}$ & $\begin{array}{r}0.064 \\
(0.60)\end{array}$ & & $\begin{array}{l}0.058 \\
(0.57)\end{array}$ \\
\hline NONDUR_SHARE & $\begin{array}{c}0.083 \\
(0.044)\end{array}$ & $\begin{array}{r}0.113 \\
(2.58)\end{array}$ & $\begin{array}{l}-0.074 \\
(0.81)\end{array}$ & $\begin{array}{r}0.003 \\
(0.04)\end{array}$ & $\begin{array}{l}-0.025 \\
(0.31)\end{array}$ & & $\begin{array}{r}2.5 \mathrm{E}-4 \\
(0.003)\end{array}$ \\
\hline MINING_SHARE & $\begin{array}{c}0.014 \\
(0.023)\end{array}$ & $\begin{array}{l}0.076 \\
(1.02)\end{array}$ & $\begin{array}{r}0.007 \\
(0.06)\end{array}$ & $\begin{array}{l}-0.144 \\
(0.99)\end{array}$ & $\begin{array}{l}-0.061 \\
(0.41)\end{array}$ & & $\begin{array}{l}-0.177 \\
(1.21)\end{array}$ \\
\hline CONST_SHARE & $\begin{array}{c}0.049 \\
(0.013)\end{array}$ & $\begin{array}{l}0.530 \\
(6.52)\end{array}$ & $\begin{array}{r}0.795 \\
(8.27)\end{array}$ & $\begin{array}{r}0.844 \\
(7.49)\end{array}$ & $\begin{array}{r}0.776 \\
(6.68)\end{array}$ & & $\begin{array}{l}0.890 \\
(7.82)\end{array}$ \\
\hline TRANPU_SHARE & $\begin{array}{c}0.053 \\
(0.010)\end{array}$ & $\begin{array}{l}-0.070 \\
(0.52)\end{array}$ & $\begin{array}{l}-0.828 \\
(3.44)\end{array}$ & $\begin{array}{l}-0.719 \\
(2.64)\end{array}$ & $\begin{array}{l}-0.863 \\
(3.07)\end{array}$ & & $\begin{array}{l}-0.587 \\
(2.13)\end{array}$ \\
\hline FIRE_SHARE & $\begin{array}{c}0.051 \\
(0.012)\end{array}$ & $\begin{array}{l}-0.169 \\
(1.75)\end{array}$ & $\begin{array}{l}-1.46 \\
(8.20)\end{array}$ & $\begin{array}{l}-1.11 \\
(5.53)\end{array}$ & $\begin{array}{l}-1.29 \\
(6.20)\end{array}$ & & $\begin{array}{l}-1.05 \\
(5.16)\end{array}$ \\
\hline TRADE_SHARE & $\begin{array}{c}0.224 \\
(0.020)\end{array}$ & $\begin{array}{r}0.108 \\
(1.44)\end{array}$ & $\begin{array}{l}-0.024 \\
(0.17)\end{array}$ & $\begin{array}{r}0.101 \\
(0.74)\end{array}$ & $\begin{array}{r}0.061 \\
(0.43)\end{array}$ & & $\begin{array}{l}0.110 \\
(0.80)\end{array}$ \\
\hline SERVICE_SHARE & $\begin{array}{c}0.222 \\
(0.046)\end{array}$ & $\begin{array}{l}0.085 \\
(1.55)\end{array}$ & $\begin{array}{r}0.187 \\
(1.81)\end{array}$ & $\begin{array}{r}0.451 \\
(4.46)\end{array}$ & $\begin{array}{r}0.481 \\
(4.59)\end{array}$ & & $\begin{array}{l}0.510 \\
(4.99)\end{array}$ \\
\hline CHANGE_FARM & $\begin{array}{l}-2.5 \mathrm{E}-4 \\
(0.002)\end{array}$ & $\begin{array}{r}0.238 \\
(1.21)\end{array}$ & $\begin{array}{r}0.195 \\
(0.97)\end{array}$ & $\begin{array}{r}0.159 \\
(0.91)\end{array}$ & $\begin{array}{r}0.142 \\
(0.78)\end{array}$ & $\begin{array}{l}-0.002 \\
(0.01)\end{array}$ & $\begin{array}{l}0.174 \\
(1.00)\end{array}$ \\
\hline CHANGE_MILITARY & $\begin{array}{r}6.0 \mathrm{E}-4 \\
(0.005)\end{array}$ & $\begin{array}{r}0.012 \\
(0.11)\end{array}$ & $\begin{array}{l}-0.137 \\
(1.22)\end{array}$ & $\begin{array}{l}-0.098 \\
(0.82)\end{array}$ & $\begin{array}{l}-0.139 \\
(1.12)\end{array}$ & $\begin{array}{l}-0.178 \\
(1.37)\end{array}$ & $\begin{array}{l}-0.104 \\
(0.87)\end{array}$ \\
\hline REGION_GROWTH & $\begin{array}{c}0.001 \\
(0.013)\end{array}$ & $\begin{array}{r}0.312 \\
(3.80)\end{array}$ & $\begin{array}{l}0.226 \\
(3.50)\end{array}$ & $\begin{array}{r}0.233 \\
(3.83)\end{array}$ & $\begin{array}{r}0.229 \\
(3.64)\end{array}$ & $\begin{array}{r}0.235 \\
(3.53)\end{array}$ & $\begin{array}{l}0.223 \\
(3.65)\end{array}$ \\
\hline REGION_GROWTH_LAG & $\begin{array}{c}0.002 \\
(0.013)\end{array}$ & $\begin{array}{r}0.103 \\
(1.41)\end{array}$ & $\begin{array}{r}0.093 \\
(1.40)\end{array}$ & $\begin{array}{r}0.081 \\
(1.25)\end{array}$ & $\begin{array}{r}0.070 \\
(1.04)\end{array}$ & $\begin{array}{r}0.102 \\
(1.44)\end{array}$ & $\begin{array}{l}0.075 \\
(1.16)\end{array}$ \\
\hline \multicolumn{8}{|l|}{$\begin{array}{l}\text { EXTERNALITY } \\
\text { VARIABLES: }\end{array}$} \\
\hline HIGH-TECH_SHARE & $\begin{array}{c}7.37 \\
(3.70)\end{array}$ & $\begin{array}{r}0.001 \\
(2.58)\end{array}$ & $\begin{array}{l}-0.003 \\
(2.69)\end{array}$ & $\begin{array}{l}-0.001 \\
(1.13)\end{array}$ & $\begin{array}{l}-0.002 \\
(1.31)\end{array}$ & $\begin{array}{l}4.9 \mathrm{E}-4 \\
(0.62)\end{array}$ & $\begin{array}{l}-0.001 \\
(0.97)\end{array}$ \\
\hline HERFINDAHL & $\begin{array}{l}578.4 \\
(96.8)\end{array}$ & $\begin{array}{l}4.0 \mathrm{E}-5 \\
(2.79)\end{array}$ & $\begin{array}{l}-5.3 \mathrm{E}-5 \\
(2.36)\end{array}$ & $\begin{array}{l}-4.4 \mathrm{E}-5 \\
(1.70)\end{array}$ & $\begin{array}{l}-6.1 \mathrm{E}-5 \\
(2.27)\end{array}$ & $\begin{array}{l}5.1 \mathrm{E}-5 \\
(2.14)\end{array}$ & $\begin{array}{l}-4.3 \mathrm{E}-5 \\
(1.64)\end{array}$ \\
\hline FIRMSIZE & $\begin{array}{l}14.32 \\
(2.51)\end{array}$ & $\begin{array}{l}-0.001 \\
(2.12)\end{array}$ & $\begin{array}{l}-0.001 \\
(2.26)\end{array}$ & $\begin{array}{l}-1.2 E-4 \\
(0.19)\end{array}$ & $\begin{array}{l}9.2 \mathrm{E}-5 \\
(0.14)\end{array}$ & $\begin{array}{l}4.0 \mathrm{E}-4 \\
(0.58)\end{array}$ & $\begin{array}{l}-1.3 \mathrm{E}-4 \\
(0.20)\end{array}$ \\
\hline METRO & $\begin{array}{c}61.25 \\
(23.58)\end{array}$ & $\begin{array}{l}1.3 \mathrm{E}-5 \\
(0.19)\end{array}$ & $\begin{array}{l}-6.6 \mathrm{E}-4 \\
(4.23)\end{array}$ & $\begin{array}{l}-2.1 \mathrm{E}-5 \\
(0.15)\end{array}$ & $\begin{array}{l}-4.5 \mathrm{E}-5 \\
(0.31)\end{array}$ & $\begin{array}{l}-5.8 \mathrm{E}-5 \\
(0.40)\end{array}$ & $\begin{array}{l}2.0 \mathrm{E}-6 \\
(0.01)\end{array}$ \\
\hline LN_SMSA_POP & $\begin{array}{c}6.65 \\
(1.21)\end{array}$ & $\begin{array}{r}0.006 \\
(4.25)\end{array}$ & na & na & na & na & na \\
\hline \multicolumn{8}{|l|}{$\cos \mathrm{T}:$} \\
\hline UNION\% & $\begin{array}{l}18.34 \\
(7.77)\end{array}$ & $\begin{array}{l}-8.6 \mathrm{E}-6 \\
(0.06)\end{array}$ & $\begin{array}{l}2.3 \mathrm{E}-4 \\
(0.83)\end{array}$ & $\begin{array}{l}3.2 \mathrm{E}-4 \\
(1.05)\end{array}$ & $\begin{array}{l}3.5 \mathrm{E}-4 \\
(1.10)\end{array}$ & $\begin{array}{l}-1.2 \mathrm{E}-4 \\
(0.39)\end{array}$ & $\begin{array}{l}5.2 \mathrm{E}-4 \\
(1.68)\end{array}$ \\
\hline
\end{tabular}


TABLE 2 (Continued)

State Competitiveness Employment Regression Results ${ }^{\mathrm{a}, \mathrm{b}}$

\begin{tabular}{|c|c|c|c|c|c|c|c|}
\hline VARIABLE & $\begin{array}{c}(1) \\
\text { MEAN } \\
\end{array}$ & $\begin{array}{l}\text { (2) } \\
\text { OLS }\end{array}$ & $\begin{array}{c}\text { (3) } \\
\text { PANEL } \\
\end{array}$ & $\begin{array}{c}\text { (4) } \\
\text { AR1 } \\
\text { PANEL } \\
\end{array}$ & $\begin{array}{c}(5) \\
\text { AR1 } \\
\text { PANEL } \\
\end{array}$ & $\begin{array}{c}(6) \\
\text { AR1 } \\
\text { PANEL } \\
\end{array}$ & $\begin{array}{c}(7) \\
2 S L S \\
\quad \text { AR1 } \\
\end{array}$ \\
\hline WAGE_MIX & $\begin{array}{c}0.991 \\
(0.039)\end{array}$ & $\begin{array}{l}-0.031 \\
(0.66)\end{array}$ & $\begin{array}{l}0.236 \\
(2.94)\end{array}$ & $\begin{array}{l}0.308 \\
(3.90)\end{array}$ & $\begin{array}{c}0.398 \\
(4.92)\end{array}$ & $\begin{array}{r}0.280 \\
(4.44)\end{array}$ & $\begin{array}{c}0.352 \\
(4.42)\end{array}$ \\
\hline WAGE_COMP & $\begin{array}{c}0.943 \\
(0.094)\end{array}$ & $\begin{array}{l}-0.090 \\
(4.37)\end{array}$ & $\begin{array}{l}-0.162 \\
(5.49)\end{array}$ & $\begin{array}{l}-0.156 \\
(4.93)\end{array}$ & $\begin{array}{l}-0.135 \\
(4.13)\end{array}$ & $\begin{array}{l}-0.093 \\
(2.98)\end{array}$ & $\begin{array}{l}-0.221 \\
(6.44)\end{array}$ \\
\hline FUEL_COST & $\begin{array}{c}0.965 \\
(0.167)\end{array}$ & $\begin{array}{l}-0.019 \\
(3.02)\end{array}$ & $\begin{array}{l}-0.036 \\
(4.22)\end{array}$ & $\begin{array}{l}-0.034 \\
(3.71)\end{array}$ & $\begin{array}{l}-0.038 \\
(3.98)\end{array}$ & $\begin{array}{l}-0.035 \\
(3.53)\end{array}$ & $\begin{array}{l}-0.032 \\
(3.45)\end{array}$ \\
\hline UI_BENEFIT & $\begin{array}{l}120.08 \\
(17.91)\end{array}$ & $\begin{array}{l}-2.4 \mathrm{E}-4 \\
(4.79)\end{array}$ & $\begin{array}{l}-3.2 \mathrm{E}-4 \\
(6.11)\end{array}$ & $\begin{array}{l}-2.9 \mathrm{E}-4 \\
(4.72)\end{array}$ & $\begin{array}{l}-3.2 E-4 \\
(4.98)\end{array}$ & $\begin{array}{l}-3.3 E-4 \\
(4.94)\end{array}$ & $\begin{array}{l}-2.4 \mathrm{E}-4 \\
(3.76)\end{array}$ \\
\hline UI_COVERAGE & $\begin{array}{l}84.88 \\
(9.02)\end{array}$ & $\begin{array}{l}-9.2 \mathrm{E}-4 \\
(6.46)\end{array}$ & $\begin{array}{l}-6.0 \mathrm{E}-4 \\
(4.69)\end{array}$ & $\begin{array}{l}-5.4 \mathrm{E}-4 \\
(4.09)\end{array}$ & $\begin{array}{l}-7.0 \mathrm{E}-4 \\
(5.16)\end{array}$ & $\begin{array}{l}-9.0 \mathrm{E}-4 \\
(6.49)\end{array}$ & $\begin{array}{l}-5.5 \mathrm{E}-4 \\
(4.10)\end{array}$ \\
\hline TAX_\%INCOME & $\begin{array}{l}10.33 \\
(1.50)\end{array}$ & $\begin{array}{l}-0.002 \\
(2.03)\end{array}$ & $\begin{array}{l}-0.003 \\
(3.74)\end{array}$ & $\begin{array}{l}-0.002 \\
(2.97)\end{array}$ & $\begin{array}{l}-0.003 \\
(4.26)\end{array}$ & $\begin{array}{l}-0.004 \\
(4.57)\end{array}$ & $\begin{array}{l}-0.002 \\
(2.88)\end{array}$ \\
\hline WELFARE_\%INCOME & $\begin{array}{c}1.92 \\
(0.64)\end{array}$ & $\begin{array}{r}0.001 \\
(0.81)\end{array}$ & $\begin{array}{l}-0.006 \\
(3.14)\end{array}$ & $\begin{array}{l}-0.005 \\
(2.36)\end{array}$ & $\begin{array}{l}-0.005 \\
(2.31)\end{array}$ & $\begin{array}{l}-0.005 \\
(2.17)\end{array}$ & $\begin{array}{l}-0.005 \\
(2.36)\end{array}$ \\
\hline \multicolumn{8}{|l|}{ DEMOGRAPHICS: } \\
\hline MALE_LABOR_FORCE\% & $\begin{array}{l}61.20 \\
(4.90)\end{array}$ & $\begin{array}{l}-0.001 \\
(5.33)\end{array}$ & $\begin{array}{l}-0.001 \\
(5.16)\end{array}$ & $\begin{array}{l}-0.001 \\
(4.57)\end{array}$ & $\begin{array}{l}-0.001 \\
(4.64)\end{array}$ & $\begin{array}{l}-0.002 \\
(6.65)\end{array}$ & $\begin{array}{l}-0.001 \\
(4.55)\end{array}$ \\
\hline BLACK_POP\% & $\begin{array}{c}9.50 \\
(9.25)\end{array}$ & $\begin{array}{l}4.4 E-5 \\
(0.20)\end{array}$ & $\begin{array}{l}-1.4 \mathrm{E}-4 \\
(0.10)\end{array}$ & $\begin{array}{l}-7.2 \mathrm{E}-4 \\
(1.49)\end{array}$ & $\begin{array}{l}-8.3 \mathrm{E}-4 \\
(1.62)\end{array}$ & $\begin{array}{l}-4.7 \mathrm{E}-4 \\
(0.97)\end{array}$ & $\begin{array}{l}-6.8 \mathrm{E}-4 \\
(1.42)\end{array}$ \\
\hline MARRIED\% & $\begin{array}{l}55.31 \\
(4.13)\end{array}$ & $\begin{array}{r}0.001 \\
(1.74)\end{array}$ & $\begin{array}{l}0.003 \\
(3.14)\end{array}$ & $\begin{array}{c}0.002 \\
(2.27)\end{array}$ & $\begin{array}{c}0.002 \\
(2.11)\end{array}$ & $\begin{array}{c}0.003 \\
(2.36)\end{array}$ & $\begin{array}{l}0.003 \\
(2.54)\end{array}$ \\
\hline FEM_MAR_CHILDU6 & $\begin{array}{l}11.70 \\
(3.73)\end{array}$ & $\begin{array}{l}-4.2 \mathrm{E}-04 \\
(0.75)\end{array}$ & $\begin{array}{l}-0.002 \\
(3.35)\end{array}$ & $\begin{array}{l}-6.0 \mathrm{E}-4 \\
(0.77)\end{array}$ & $\begin{array}{l}-6.5 E-4 \\
(0.80)\end{array}$ & $\begin{array}{l}-0.001 \\
(1.43)\end{array}$ & $\begin{array}{l}-2.2 \mathrm{E}-4 \\
(0.27)\end{array}$ \\
\hline FEM_NONMAR_CHILDU6 & $\begin{array}{c}1.62 \\
(0.48)\end{array}$ & $\begin{array}{l}0.002 \\
(0.94)\end{array}$ & $\begin{array}{l}-0.003 \\
(0.97)\end{array}$ & $\begin{array}{r}1.2 \mathrm{E}-5 \\
(0.004)\end{array}$ & $\begin{array}{l}-0.002 \\
(0.68)\end{array}$ & $\begin{array}{l}-0.002 \\
(0.70)\end{array}$ & $\begin{array}{l}-9.7 \mathrm{E}-4 \\
(0.31)\end{array}$ \\
\hline AGE_OVER64\% & $\begin{array}{c}0.118 \\
(0.019)\end{array}$ & $\begin{array}{l}-0.218 \\
(2.79)\end{array}$ & $\begin{array}{r}0.130 \\
(0.73)\end{array}$ & $\begin{array}{r}0.079 \\
(0.42)\end{array}$ & $\begin{array}{c}0.084 \\
(0.43)\end{array}$ & $\begin{array}{c}0.397 \\
(2.36)\end{array}$ & $\begin{array}{l}-0.085 \\
(0.44)\end{array}$ \\
\hline AGE_UNDER 15\% & $\begin{array}{c}0.233 \\
(0.027)\end{array}$ & $\begin{array}{l}-0.184 \\
(2.87)\end{array}$ & $\begin{array}{l}-0.524 \\
(4.17)\end{array}$ & $\begin{array}{l}-0.348 \\
(2.52)\end{array}$ & $\begin{array}{l}-0.411 \\
(2.87)\end{array}$ & $\begin{array}{l}-0.248 \\
(1.89)\end{array}$ & $\begin{array}{l}-0.451 \\
(3.21)\end{array}$ \\
\hline INTERNATIONAL_MIG & $\begin{array}{c}0.001 \\
(0.002)\end{array}$ & $\begin{array}{c}1.02 \\
(2.22)\end{array}$ & $\begin{array}{r}0.357 \\
(0.87)\end{array}$ & $\begin{array}{c}0.274 \\
(0.62)\end{array}$ & $\begin{array}{l}-0.086 \\
(0.19)\end{array}$ & $\begin{array}{r}0.505 \\
(1.04)\end{array}$ & $\begin{array}{c}0.385 \\
(0.86)\end{array}$ \\
\hline HIGHSCHOOL_GRAD & $\begin{array}{l}67.27 \\
(9.33)\end{array}$ & $\begin{array}{l}9.7 \mathrm{E}-4 \\
(4.39)\end{array}$ & $\begin{array}{l}-3.4 \mathrm{E}-4 \\
(0.70)\end{array}$ & $\begin{array}{l}4.6 \mathrm{E}-4 \\
(0.92)\end{array}$ & $\begin{array}{l}7.1 \mathrm{E}-4 \\
(1.37)\end{array}$ & $\begin{array}{l}7.3 \mathrm{E}-4 \\
(1.47)\end{array}$ & $\begin{array}{l}3.8 \mathrm{E}-4 \\
(0.76)\end{array}$ \\
\hline COLLEGE_GRAD & $\begin{array}{l}16.12 \\
(3.94)\end{array}$ & $\begin{array}{l}-0.001 \\
(2.06)\end{array}$ & $\begin{array}{l}-0.002 \\
(2.11)\end{array}$ & $\begin{array}{l}-0.005 \\
(4.39)\end{array}$ & $\begin{array}{l}-0.006 \\
(5.09)\end{array}$ & $\begin{array}{l}-0.004 \\
(3.77)\end{array}$ & $\begin{array}{l}-0.005 \\
(4.13)\end{array}$ \\
\hline REGION DUMMIES $^{c}$ & & YES & na & na & na & na & na \\
\hline $\begin{array}{l}0001 \text { = F-TEST: One-Digit } \\
\text { Employment Shares }^{\mathrm{d}}\end{array}$ & & $\begin{array}{c}8.55 \\
(p=.0001)\end{array}$ & $\begin{array}{c}19.64 \\
(p=.0001)\end{array}$ & $\begin{array}{c}14.85 \\
(p=.0001)\end{array}$ & $\begin{array}{c}14.43 \\
(p=.0001)\end{array}$ & na & $\begin{array}{c}15.45 \\
(p=.0001)\end{array}$ \\
\hline $\mathbf{R}^{2}$ & & 0.52 & 0.66 & 0.57 & 0.54 & 0.45 & 0.55 \\
\hline
\end{tabular}

The dependent variable of the regression is the 1972-1991 state competitiveness employment growth rate.

b In parentheses are standard deviations in column (1) and absolute values of the t-statistics in the remaining columns. The OLS results use the White-heteroskedasticity-consistent t-statistics.

"Nine region dummy variables.

${ }^{\text {d}}$ F-statistic for the joint null hypothesis that the one-digit employment share coefficients (e.g., for

FARM_SHARE, DUR_SHARE, etc.) are all equal to zero. P-values are in parentheses. 
creates additional jobs in the state's economy through a traditional employment multiplier effect (DEMAND in Equation (7)).

To account for other exogenous (or disequilibrium) demand shifts, the annual change in the farm employment share (CHANGE_FARM) and the change in military spending as a share of gross state product (CHANGE_MILITARY) are also included. To account for employment growth that is induced by economic growth in surrounding states, employment growth in the four Census regions net of the state's employment growth and its lag are included as additional variables (REGION_GROWTH, REGION_GROWTH_LAG).

Factors that affect $\mathrm{h}($.$) in Equation (7) include one-digit employment shares$ in farming (FARM_SHARE); durable goods manufacturing (DUR_SHARE); nondurable goods manufacturing (NONDUR_SHARE); mining (MINING_SHARE); construction (CONST_SHARE); transportation and public utilities (TRANPU_SHARE); finance, insurance, and real estate (FIRE_SHARE); wholesale and retail trade (TRADE_SHARE); and services (SERVICE_SHARE). Government is the omitted sector, so the industry share coefficients should be interpreted as being relative to the government sector.

Since the direct industry mix effect on employment growth is already accounted for, the industry share terms control for the interrelationships between sectors (e.g., Garcia-Milà and McGuire, 1993). For the most part, the share terms should account for each sector's influence due to their roles as demanders of inputs from other industries and as suppliers of inputs to other industries that are not captured on average by the estimated INDMIX_EMP coefficients. For example, if sector A purchases a relatively large share of its inputs locally, the impact of sector A on state employment would be greater than what is suggested by the employment effect implied by the INDMIX_EMP coefficients. Thus, states with a larger share of sector A will grow faster, holding the state's industrial mix employment growth rate constant. In addition, the industry share terms should control for productivity differences between sectors. Also, the manufacturing employment share variable controls for whether the region is relatively underserved in manufacturing, which in turn may result in new plants locating in the state.

Several variables are included to measure the impact of knowledge spillovers stressed in endogenous growth theory. The first variable is the percent of private nonfarm employment in high-tech manufacturing (HIGH-TECH_SHARE). ${ }^{7}$ HIGH-TECH_SHARE controls for the possible positive influence of R\&D spillovers, knowledge spillovers, or even subtle demonstration effects that high-tech firms could have on neighboring firms. Thus, the inclusion of $\mathrm{HIGH}$ TECH_SHARE directly tests whether having a concentration of R\&D-intensive high-tech firms induces additional employment growth in a state. Conversely, if 
high-tech firms disproportionately purchase inputs from other regions or internationally, HIGH-TECH_SHARE could be negatively related to employment growth, suggesting that the HIGH-TECH_SHARE coefficient's sign is ambiguous.

Two measures of market structure are included. The first is a two-digit Herfindahl index of employment concentration. The Herfindahl index measures whether concentrations of industries result in within-industry knowledge spillovers as suggested by the endogenous growth models. Alternatively, it could be that spillovers occur more between industries rather than between firms in an industry suggesting that a more diverse economy would spur growth (Glaeser et al. 1992). Thus, HERFINDAHL has an ambiguous impact. Second, average nonfarm private sector establishment size in the state is also included in the model (FIRMSIZE). Larger monopoly firms may have more incentive for innovation because of smaller appropriability concerns (e.g., Jorde and Teece, 1990). Conversely, smaller competitive firms have more market pressure to innovate, suggesting an ambiguous effect (e.g., Porter, 1990). ${ }^{8}$

We control for urbanization effects by including the percent of the state's population that lives in a metropolitan area (METRO). Knowledge spillovers and agglomeration effects are believed to frequently occur in urban areas (e.g., Lucas, 1988). This suggests that urbanization effects are the reason that major metropolitan economies support high land rents and other costs. Nonetheless, after a certain threshold, the benefits of increased urbanization could be offset by greater production costs. In the OLS specifications, we also include as a fixed effect the natural log of the 1972 population of the state's largest metropolitan area ( $L N \_S M S A \_P O P$ ). $L N \_S M S A \_P O P$ tests whether states that began the period with a large city were more conducive to economic activity.

Several variables are included to measure the influence of cost and state business climate differences on labor demand. A major component of relative costs is how the state's relative wage structure compares to other states. Two measures of the state's relative wage structure are derived from annual nonfarm private wages to capture disequilibrium adjustments to differing state cost structures. First, a measure of whether the state's mix of industries is relatively highpaying is calculated (WAGE_MIX). More formally, WAGE_MIX is the state-employment-weighted average of corresponding national industry wage rates expressed relative to the average U.S. private wage rate (U.S. Bureau of Labor Statistics). A greater WAGE_MIX ratio is hypothesized to positively shift labor demand through the spillover or multiplier effects of having a larger proportion of high-paying industries. Second, WAGE_COMP is calculated as the ratio of the actual state wage rate divided by state-employment-weighted U.S. industry wage rates. ${ }^{9}$ Holding the state's average wage fixed, the WAGE_COMP ratio 
measures whether the state's wages are relatively high after taking its industry mix into account. A higher WAGE_COMP indicates that the typical industry in the state is paying higher wages than the industry's national average, which suggests that the typical industry in the state may be at a competitive disadvantage. Thus, a greater WAGE_COMP ratio should reduce labor demand.

An index of the state's fuel costs (FUEL_COST) is another control for a state's cost structure. Higher fuel costs reduce employment growth through adverse competitiveness effects but increase employment growth through substitution of labor for fuel in production, producing a theoretically ambiguous effect.

Another aspect of the labor climate is the percent of the nonagricultural labor force that is unionized (UNION\%). Aside from the greater wage costs that are already controlled for, a more unionized labor force can increase the number of rigid work rules, which reduces employment in more unionized states. Conversely, there could be offsetting union voice effects that enhance union productivity (e.g., Freeman and Medoff, 1984), which increase employment.

Several measures of the government business climate are included in the model. First, the percent of state and local taxes as a share of personal income is included (TAX_\%INCOME). Taxes are an important control because some taxes directly increase costs, and to some firms, lower taxes reflect a state government's commitment to the business community. Moreover, higher household taxes may cause out-migration or deter potential in-migrants. However, offsetting the negative influence of taxes is the positive influence of some of the services that government provides. Other policy variables that affect production costs include the availability and generosity of unemployment insurance (UI_COVERAGE, UI_BENEFIT) and public welfare as a percent of personal income (WELFARE_\%INCOME). Liberal unemployment benefits can increase business payroll taxes or increase the reservation wage of the labor force, both of which can retard employment growth.

The percent of the population above the age of 24 who are high school graduates (HIGHSCHOOL_GRAD) and who are college graduates (COLLEGE_GRAD) are included to control for the human capital of the laborforce. If the average level of human capital in the labor force is associated with increased economic growth (Lucas, 1988; Rauch, 1993), it is expected that both educational attainment coefficients will be positive.

Demographic characteristics are also included as control variables. The percent of the labor force that is male (MALE_LABOR_FORCE\%) is included to control for the effects of differential changes in female participation rates. To control for possible discrimination, labor force quality, and preferences for work, the percent of the population that is black (BLACKPOP\%); 14 years or younger (AGE_UNDER15\%); 65 years or older (AGE_OVER64\%); married 
(MARRIED\%); married women that have children six years old or younger (FEM_MAR_CHILDU6); and unmarried women that have children six years old or younger (FEM_NONMAR_CHILDU6) are included. New international migrants (INTERNATIONAL_MIG), as a share of the population, are included to control for exogenous labor supply shifts that would increase relative employment growth. Alternatively, out-migration of less skilled natives from states exposed to more international migration (Frey, 1994) may offset the outward labor supply shift associated with international migration. Moreover, recent social tensions surrounding increased international immigration (e.g., in California and Florida) suggest that greater international immigration may reduce economic growth.

\section{EMPIRICAL RESULTS}

Table 2 presents the competitiveness employment growth results, while Table 3 presents the results for state employment growth relative to the nation. Because the results are quite similar, most of the emphasis will be on the competitiveness employment growth results. In Table 2, Column (2) shows the ordinary least square results. Dummies for the nine major Census divisions are included in this model (OLS) to account for regional fixed effects $\left(\sigma_{\mathrm{r}}\right)$.

Summing the coefficients for INDMIX_EMP and INDMIX_EMP_LAG suggests that a two-year 1 percent increase in the industry mix growth rate spurs an additional 1.3 percent increase in the competitive employment growth rate by the second year. These results suggest large employment multiplier effects of having a mix of industries growing faster than the national average. Moreover, the results suggest that there are fairly significant economic spillovers from neighboring states in the region. Specifically, by summing the REGION_GROWTH and REGION_GROWTH_LAG coefficients, a two-year 1 percent increase in employment growth in other states in the region increases that state's economic growth by a little over 0.4 percent by the second year. Finally, states that have larger shares of employment in durable goods manufacturing, nondurable goods manufacturing, and construction have greater employment growth. Thus, the OLS results support the traditional assumption of state and local economic development policymakers-overall employment multipliers are quite large and development efforts should focus on manufacturing. ${ }^{10}$

The OLS results are somewhat consistent with various strands of the endogenous growth literature. Even after controlling for indirect impacts through wages and employment, states with a larger share of employment in R\&D intensive high-tech manufacturing had a greater competitiveness employment growth rate. Similarly, states with greater concentrations of particular industries, as 
TABLE 3

Relative Total State Employment Regression Results ${ }^{\mathrm{a}, \mathrm{b}}$

\begin{tabular}{|c|c|c|c|c|c|}
\hline VARIABLE & $\begin{array}{l}\text { (1) } \\
\text { OLS }\end{array}$ & $\begin{array}{c}\text { (2) } \\
\text { PANEL }\end{array}$ & $\begin{array}{c}\text { (3) } \\
\text { AR1 } \\
\text { PANEL }\end{array}$ & $\begin{array}{c}(4) \\
\text { AR1 } \\
\text { PANEL }\end{array}$ & $\begin{array}{c}(5) \\
\text { 2SLS } \\
\text { AR1 }\end{array}$ \\
\hline \multicolumn{6}{|c|}{ DEMAND OR STRUCTURAL: } \\
\hline FARM_SHARE & $\begin{array}{c}0.243 \\
(1.97)\end{array}$ & $\begin{array}{c}0.088 \\
(0.43)\end{array}$ & $\begin{array}{l}-0.112 \\
(0.50)\end{array}$ & & $\begin{array}{l}-0.097 \\
(0.43)\end{array}$ \\
\hline DUR_SHARE & $\begin{array}{c}0.160 \\
(2.85)\end{array}$ & $\begin{array}{c}0.304 \\
(2.60)\end{array}$ & $\begin{array}{r}0.071 \\
(0.58)\end{array}$ & & $\begin{array}{c}0.055 \\
(0.45)\end{array}$ \\
\hline NONDUR_SHARE & $\begin{array}{c}0.088 \\
(1.64)\end{array}$ & $\begin{array}{c}0.007 \\
(0.07)\end{array}$ & $\begin{array}{l}-0.044 \\
(0.47)\end{array}$ & & $\begin{array}{l}-0.050 \\
(0.53)\end{array}$ \\
\hline MINING_SHARE & $\begin{array}{c}0.133 \\
(1.37)\end{array}$ & $\begin{array}{r}0.187 \\
(1.33)\end{array}$ & $\begin{array}{c}0.002 \\
(0.01)\end{array}$ & & $\begin{array}{l}-0.029 \\
(0.18)\end{array}$ \\
\hline CONST_SHARE & $\begin{array}{r}0.582 \\
(6.06)\end{array}$ & $\begin{array}{c}0.775 \\
(7.10)\end{array}$ & $\begin{array}{c}0.701 \\
(5.46)\end{array}$ & & $\begin{array}{c}0.736 \\
(5.69)\end{array}$ \\
\hline TRANPU_SHARE & $\begin{array}{l}-0.045 \\
(0.29)\end{array}$ & $\begin{array}{l}-0.840 \\
(3.08)\end{array}$ & $\begin{array}{l}-0.977 \\
(3.11)\end{array}$ & & $\begin{array}{l}-0.869 \\
(2.75)\end{array}$ \\
\hline FIRE_SHARE & $\begin{array}{l}-0.097 \\
(0.87)\end{array}$ & $\begin{array}{l}-1.81 \\
(9.01)\end{array}$ & $\begin{array}{l}-1.50 \\
(6.47)\end{array}$ & & $\begin{array}{l}-1.45 \\
(6.24)\end{array}$ \\
\hline TRADE_SHARE & $\begin{array}{c}0.163 \\
(1.77)\end{array}$ & $\begin{array}{r}0.044 \\
(0.28)\end{array}$ & $\begin{array}{l}-0.016 \\
(0.10)\end{array}$ & & $\begin{array}{l}-0.013 \\
(0.08)\end{array}$ \\
\hline SERVICE_SHARE & $\begin{array}{c}0.153 \\
(2.12)\end{array}$ & $\begin{array}{c}0.368 \\
(3.19)\end{array}$ & $\begin{array}{r}0.501 \\
(4.20)\end{array}$ & & $\begin{array}{r}0.544 \\
(4.52)\end{array}$ \\
\hline CHANGE_FARM & $\begin{array}{r}0.139 \\
(0.58)\end{array}$ & $\begin{array}{r}0.091 \\
(0.40)\end{array}$ & $\begin{array}{c}0.113 \\
(0.54)\end{array}$ & $\begin{array}{l}-0.040 \\
(0.22)\end{array}$ & $\begin{array}{c}0.125 \\
(0.60)\end{array}$ \\
\hline CHANGE_MILITARY & $\begin{array}{l}-0.095 \\
(0.83)\end{array}$ & $\begin{array}{l}-0.234 \\
(1.84)\end{array}$ & $\begin{array}{l}-0.161 \\
(1.16)\end{array}$ & $\begin{array}{l}-0.167 \\
(1.15)\end{array}$ & $\begin{array}{l}-0.163 \\
(1.17)\end{array}$ \\
\hline REGION_GROWTH & $\begin{array}{c}0.308 \\
(3.19)\end{array}$ & $\begin{array}{c}0.212 \\
(2.89)\end{array}$ & $\begin{array}{r}0.244 \\
(3.43)\end{array}$ & $\begin{array}{c}0.258 \\
(3.47)\end{array}$ & $\begin{array}{r}0.237 \\
(3.32)\end{array}$ \\
\hline REGION_GROWTH_LAG & $\begin{array}{c}0.063 \\
(0.73)\end{array}$ & $\begin{array}{r}0.078 \\
(1.04)\end{array}$ & $\begin{array}{r}0.047 \\
(0.62)\end{array}$ & $\begin{array}{r}0.074 \\
(0.94)\end{array}$ & $\begin{array}{r}0.040 \\
(0.54)\end{array}$ \\
\hline EXTERNALITY VARIABI & & & & & \\
\hline HIGH-TECH_SHARE & $\begin{array}{r}0.001 \\
(2.08)\end{array}$ & $\begin{array}{l}-0.004 \\
(3.51)\end{array}$ & $\begin{array}{l}-0.002 \\
(1.52)\end{array}$ & $\begin{array}{c}5.1 \mathrm{E}-4 \\
(0.59)\end{array}$ & $\begin{array}{l}-0.002 \\
(1.38)\end{array}$ \\
\hline HERFINDAHL & $\begin{array}{l}3.8 \mathrm{E}-5 \\
(2.30)\end{array}$ & $\begin{array}{l}-8.4 \mathrm{E}-5 \\
(3.33)\end{array}$ & $\begin{array}{l}-7.6 \mathrm{E}-5 \\
(2.55)\end{array}$ & $\begin{array}{l}3.9 \mathrm{E}-5 \\
(1.46)\end{array}$ & $\begin{array}{l}-7.5 \mathrm{E}-5 \\
(2.50)\end{array}$ \\
\hline FIRMSIZE & $\begin{array}{l}1.3 \mathrm{E}-5 \\
(0.02)\end{array}$ & $\begin{array}{l}-7.4 \mathrm{E}-4 \\
(1.09)\end{array}$ & $\begin{array}{l}3.4 \mathrm{E}-4 \\
(0.44)\end{array}$ & $\begin{array}{l}6.9 \mathrm{E}-4 \\
(0.90)\end{array}$ & $\begin{array}{l}3.4 E-4 \\
(0.44)\end{array}$ \\
\hline METRO & $\begin{array}{l}3.4 \mathrm{E}-5 \\
(0.42)\end{array}$ & $\begin{array}{l}-7.5 E-4 \\
(4.23)\end{array}$ & $\begin{array}{l}-6.7 E-5 \\
(0.39)\end{array}$ & $\begin{array}{l}-1.0 \mathrm{E}-4 \\
(0.61)\end{array}$ & $\begin{array}{l}-5.2 \mathrm{E}-5 \\
(0.31)\end{array}$ \\
\hline LN_SMSA_POP & $\begin{array}{r}0.005 \\
(2.77)\end{array}$ & na & na & na & na \\
\hline cost: & & & & & \\
\hline UNION\% & $\begin{array}{l}1.4 \mathrm{E}-4 \\
(0.76)\end{array}$ & $\begin{array}{l}3.9 \mathrm{E}-4 \\
(1.23)\end{array}$ & $\begin{array}{l}3.6 \mathrm{E}-4 \\
(1.03)\end{array}$ & $\begin{array}{l}-3.8 \mathrm{E}-5 \\
(0.11)\end{array}$ & $\begin{array}{c}5.2 \mathrm{E}-4 \\
(1.47)\end{array}$ \\
\hline WAGE_MIX & $\begin{array}{l}-0.017 \\
(0.28)\end{array}$ & $\begin{array}{c}0.444 \\
(4.96)\end{array}$ & $\begin{array}{r}0.496 \\
(5.44)\end{array}$ & $\begin{array}{r}0.372 \\
(5.29)\end{array}$ & $\begin{array}{r}0.538 \\
(5.84)\end{array}$ \\
\hline WAGE_COMP & $\begin{array}{l}-0.060 \\
(2.50)\end{array}$ & $\begin{array}{l}-0.144 \\
(4.29)\end{array}$ & $\begin{array}{l}-0.111 \\
(3.04)\end{array}$ & $\begin{array}{l}-0.080 \\
(2.30)\end{array}$ & $\begin{array}{l}-0.166 \\
(4.19)\end{array}$ \\
\hline FUEL_COST & $\begin{array}{l}-0.011 \\
(1.48)\end{array}$ & $\begin{array}{c}-0.040 \\
(4.17)\end{array}$ & $\begin{array}{c}-0.044 \\
(4.07)\end{array}$ & $\begin{array}{l}-0.041 \\
(3.64)\end{array}$ & $\begin{array}{l}-0.042 \\
(3.92)\end{array}$ \\
\hline UI_BENEFIT & $\begin{array}{l}-3.3 \mathrm{E}-4 \\
(5.21)\end{array}$ & $\begin{array}{l}-3.9 \mathrm{E}-4 \\
(6.49)\end{array}$ & $\begin{array}{l}-3.5 \mathrm{E}-4 \\
(4.95)\end{array}$ & $\begin{array}{l}-3.7 \mathrm{E}-4 \\
(4.95)\end{array}$ & $\begin{array}{l}-3.1 \mathrm{E}-4 \\
(4.25)\end{array}$ \\
\hline UI_COVERAGE & $\begin{array}{l}-0.001 \\
(7.67)\end{array}$ & $\begin{array}{l}-8.6 \mathrm{E}-4 \\
(6.02)\end{array}$ & $\begin{array}{l}-9.4 \mathrm{E}-4 \\
(6.07)\end{array}$ & $\begin{array}{l}-0.001 \\
(7.34)\end{array}$ & $\begin{array}{l}-9.4 \mathrm{E}-4 \\
(6.08)\end{array}$ \\
\hline TAX_\%INCOME & $\begin{array}{l}-0.002 \\
(2.00)\end{array}$ & $\begin{array}{l}-0.005 \\
(6.30)\end{array}$ & $\begin{array}{l}-0.005 \\
(5.24)\end{array}$ & $\begin{array}{l}-0.005 \\
(5.34)\end{array}$ & $\begin{array}{l}-0.005 \\
(5.20)\end{array}$ \\
\hline WELFARE_\%INCOME & $\begin{array}{r}0.003 \\
(1.62)\end{array}$ & $\begin{array}{l}-0.007 \\
(2.87)\end{array}$ & $\begin{array}{l}-0.006 \\
(2.17)\end{array}$ & $\begin{array}{l}-0.005 \\
(1.75)\end{array}$ & $\begin{array}{l}-0.006 \\
(2.18)\end{array}$ \\
\hline
\end{tabular}


TABLE 3 (Continued)

Relative Total State Employment Regression Results ${ }^{\mathrm{a}, \mathrm{b}}$

\begin{tabular}{|c|c|c|c|c|c|}
\hline VARIABLE & $\begin{array}{l}\text { (1) } \\
\text { OLS }\end{array}$ & $\begin{array}{c}\text { (2) } \\
\text { PANEL }\end{array}$ & $\begin{array}{c}\text { (3) } \\
\text { AR1 } \\
\text { PANEL }\end{array}$ & $\begin{array}{c}\text { (4) } \\
\text { AR1 } \\
\text { PANEL }\end{array}$ & $\begin{array}{c}\text { (5) } \\
2 S L S \\
\text { AR1 }\end{array}$ \\
\hline \multicolumn{6}{|l|}{ DEMOGRAPHICS: } \\
\hline MALE_LABOR_FORCE\% & $\begin{array}{l}-9.7 \mathrm{E}-4 \\
(3.74)\end{array}$ & $\begin{array}{l}-0.001 \\
(3.45)\end{array}$ & $\begin{array}{l}-0.001 \\
(4.16)\end{array}$ & $\begin{array}{l}-0.002 \\
(6.12)\end{array}$ & $\begin{array}{l}-0.001 \\
(4.16)\end{array}$ \\
\hline BLACK_POP\% & $\begin{array}{l}-1.2 \mathrm{E}-4 \\
(0.44)\end{array}$ & $\begin{array}{l}-5.2 \mathrm{E}-4 \\
(0.31)\end{array}$ & $\begin{array}{l}-8.8 \mathrm{E}-4 \\
(1.41)\end{array}$ & $\begin{array}{l}-5.8 \mathrm{E}-4 \\
(1.04)\end{array}$ & $\begin{array}{l}-8.6 \mathrm{E}-4 \\
(1.39)\end{array}$ \\
\hline MARRIED\% & $\begin{array}{l}5.0 \mathrm{E}-4 \\
(0.63)\end{array}$ & $\begin{array}{c}0.004 \\
(3.67)\end{array}$ & $\begin{array}{r}0.002 \\
(2.00)\end{array}$ & $\begin{array}{c}0.002 \\
(1.72)\end{array}$ & $\begin{array}{c}0.003 \\
(2.21)\end{array}$ \\
\hline FEM_MAR_CHILDU6 & $\begin{array}{l}3.9 \mathrm{E}-4 \\
(0.58)\end{array}$ & $\begin{array}{l}-0.002 \\
(3.06)\end{array}$ & $\begin{array}{l}-9.5 \mathrm{E}-4 \\
(1.05)\end{array}$ & $\begin{array}{l}-0.001 \\
(1.49)\end{array}$ & $\begin{array}{l}-6.4 E-4 \\
(0.71)\end{array}$ \\
\hline FEM_NONMAR_CHILDU6 & $\begin{array}{l}0.003 \\
(1.10)\end{array}$ & $\begin{array}{l}-0.005 \\
(1.63)\end{array}$ & $\begin{array}{l}-0.005 \\
(1.25)\end{array}$ & $\begin{array}{l}-0.004 \\
(1.13)\end{array}$ & $\begin{array}{l}-0.005 \\
(1.50)\end{array}$ \\
\hline AGE_OVER64\% & $\begin{array}{l}-0.065 \\
(0.74)\end{array}$ & $\begin{array}{r}0.277 \\
(1.37)\end{array}$ & $\begin{array}{c}0.133 \\
(0.60)\end{array}$ & $\begin{array}{r}0.386 \\
(2.04)\end{array}$ & $\begin{array}{l}-0.007 \\
(0.03)\end{array}$ \\
\hline AGE_UNDER15\% & $\begin{array}{l}-0.144 \\
(1.84)\end{array}$ & $\begin{array}{l}-0.540 \\
(3.78)\end{array}$ & $\begin{array}{l}-0.520 \\
(3.21)\end{array}$ & $\begin{array}{l}-0.302 \\
(2.07)\end{array}$ & $\begin{array}{l}-0.604 \\
(3.68)\end{array}$ \\
\hline INTERNATIONAL_MIG & $\begin{array}{l}0.280 \\
(0.51)\end{array}$ & $\begin{array}{l}-0.284 \\
(0.61)\end{array}$ & $\begin{array}{l}-0.422 \\
(0.83)\end{array}$ & $\begin{array}{l}0.189 \\
(0.35)\end{array}$ & $\begin{array}{l}-0.335 \\
(0.66)\end{array}$ \\
\hline HIGHSCHOOL_GRAD & $\begin{array}{r}0.001 \\
(3.90)\end{array}$ & $\begin{array}{l}3.7 \mathrm{E}-4 \\
(0.66)\end{array}$ & $\begin{array}{l}9.7 \mathrm{E}-4 \\
(1.64)\end{array}$ & $\begin{array}{l}9.8 \mathrm{E}-4 \\
(1.76)\end{array}$ & $\begin{array}{l}9.1 \mathrm{E}-4 \\
(1.54)\end{array}$ \\
\hline COLLEGE_GRAD & $\begin{array}{l}-2.1 E-4 \\
(0.34)\end{array}$ & $\begin{array}{l}-0.003 \\
(2.50)\end{array}$ & $\begin{array}{l}-0.007 \\
(5.28)\end{array}$ & $\begin{array}{l}-0.005 \\
(4.15)\end{array}$ & $\begin{array}{l}-0.007 \\
(5.09)\end{array}$ \\
\hline REGION DUMMIES $^{c}$ & YES & na & na & na & na \\
\hline F-TEST: One-Digit & 7.80 & 18.43 & 12.13 & na & 12.35 \\
\hline $\begin{array}{l}\text { Employment Shares } \\
\mathbf{R}^{2}\end{array}$ & $\begin{array}{c}(p=.0001) \\
0.45\end{array}$ & $\begin{array}{c}(p=.0001) \\
0.62\end{array}$ & $\begin{array}{c}(p=.0001) \\
0.55\end{array}$ & 0.46 & $\begin{array}{c}(p=.0001) \\
0.51\end{array}$ \\
\hline
\end{tabular}

The dependent variable of the regression is the 1972-1991 state employment growth rate minus the U.S. employment growth rate.

'In parentheses are absolute values of the t-statistics. The OLS results use the White-heteroskedasticity-consistent t-statistics.

cNine region dummy variables.

${ }^{d}$ F-statistic for the joint null hypothesis that the one-digit employment share coefficients (e.g., for FARM_SHARE, DUR_SHARE, etc.) are all equal to zero. P-values are in parentheses.

reflected through a larger Herfindahl index, have greater employment growth. Thus, the OLS results suggest that knowledge spillovers and other industry agglomeration effects are important. Finally, the OLS results suggest that there are positive urbanization effects on employment of having one large city (LN_SMSA_POP) that can attract growth. Simply having many smaller metropolitan areas (METRO), however, has little additional impact.

The OLS results suggest that both measures of the state's relative wage structure are negatively related to employment growth (WAGE_MIX, WAGE_COMP), although a state's wage mix is insignificant. Finally, the INTERNATIONAL_MIG coefficient suggests that there is almost a one-for-one employment gain with increased international migration, which suggests little net (across all groups) native out-migration offsetting the new immigration. 
One concern with the OLS results is that omitted state-fixed effects could bias the coefficients. Thus, the results in column (3) add state fixed effects to the specification. The coefficient of determination suggests that the state dummies significantly improve the explanatory power of the model. However, a problem with fixed effect specifications is that they do not utilize cross-sectional differences in the independent variables in estimating the slope coefficients, i.e., only within state time series variation is used to estimate the slope coefficients. One implication is that fixed effects models may be overly restrictive where some coefficients may incorrectly appear to be insignificant because of insufficient variation over time.

The first-order correlation of the error terms equalled 0.38 in column (3), suggesting that autocorrelation may be present. Thus, column (4) presents results of a specification that corrects for autocorrelation through a Cochrane-Orcutt-like two-step generalized differencing procedure, in which the first observation for each state is omitted during the estimation of rho in the first step. The first-order correlation of the error terms fell to 0.08 in this specification. Overall, there are only minor differences between the specifications in columns (3) and (4), but there are significant differences between the OLS results and the two fixed effects specifications. Hence, even when controlling for nine regions, omitted state fixed effects may still bias the OLS results, resulting in misleading findings. For brevity, only the differences between the OLS results in column (2) and the autocorrelation-corrected results in column (4) are highlighted.

The influence of a favorable INDMIX_EMP is smaller when state fixed effects are accounted for, but these results still suggest that a state with an industry. mix that is growing 1 percent faster than normal for two years will experience an additional 1.2 percent faster competitiveness employment growth by the second year (by summing the two INDMIX_EMP coefficients). These findings illustrate that economic development policymakers need to continuously reassess their strategies to stay focussed on nationally expanding industries because of the large spillovers on other sectors of the economy.

Although they are smaller when state fixed effects are included, employment spillovers between states remain quite large (REGION_GROWTH, REGION_GROWTH_LAG). The large employment spillovers between states suggest that instead of simply fiercely competing, neighboring states should also cooperate more in their economic development efforts.

The WAGE_MIX ratio coefficient suggests that a mix of high-paying industries increases the competitiveness employment growth rate. However, the WAGE_COMP ratio coefficient implies that if wages in the state's industries get out of line with their national norms, employment growth declines. Specifically, column (4)'s WAGE_MIX coefficient suggests that changes in the state's in- 
dustrial composition that result in a mix of industries that pay 1 percent higher average national wages increases that year's competitiveness employment growth rate by about 0.31 percent (i.e., $0.01 * 0.308$ ). Conversely, the WAGE_COMP coefficient indicates that if wages in the state's industries rise by 1 percent relative to the industries' national average, the state's competitiveness employment growth will slow by about 0.16 percent that year $(0.01 *(-0.156))$. Overall, our hypothesis that employment expands at a faster rate in states with a composition of high-paying industries is supported (i.e., WAGE_MIX), where the decomposition of total wages allowed us to identify the two separate wage effects. Moreover, given the relative size of the WAGE_MIX and WAGE_COMP coefficients, policymakers are advised to attract high-wage industries, even if the high wages from one industry induce higher wages in other industries, hurting their competitiveness.

Previous studies typically control only for wages without adjusting for the influence of industry composition. A similar control can be derived by multiplying WAGE_COMP and WAGE_MIX, which creates a measure of each state's overall wage relative to the national average wage. To examine the effect of controlling for relative wages without adjusting for industry mix, this variable was used in place of WAGE_MIX and WAGE_COMP using the model shown in column (4) (not shown). The coefficient on the relative wage variable equalled -0.09 $(t=-3.83)$, which is smaller in magnitude than the WAGE_COMP coefficient. This indicates that the previous literature tends to underestimate the impact of high wages on employment by not accounting for the effects of industry composition on wages.

F-tests at the bottom of Table 2 suggest that the one-digit employment shares are jointly statistically significant. The autocorrelation-corrected results suggest that, unlike what Garcia-Milà and McGuire (1993) found, a greater share of employment in manufacturing does not significantly influence competitiveness employment. Only construction (like $G \&$ M) and services (unlike $G \& M$ ) have significantly positive effects relative to the government sector. In addition, transportation and public utilities (like $G \& M$ ) and finance, insurance, and real estate (unlike $\mathbf{G} \& \mathbf{M}$ ) have a negative influence, while the other sectors did not significantly differ from government in their effect (e.g., manufacturing). However, our results are not directly comparable to Garcia-Milà and McGuire (1993) because our model controls for many more potential factors. ${ }^{11}$ The influence of industry composition on employment, after accounting for how industry mix affects employment and wages, suggests that industry composition has an additional role. One possible explanation is that interrelationships between industries as both demanders and suppliers of inputs depend on industry composition. 
Once fixed effects are accounted for, our knowledge spillover and agglomeration effect measures are not positively statistically significant. If anything, states with a greater share of high-tech manufacturing have smaller competitiveness employment growth rates. ${ }^{12}$ Thus, knowledge spillover effects that would arise from having a concentration of R\&D-intensive firms are apparently dominated by other factors, including the possibility that high-tech firms obtain a large share of their inputs from other regions, which would reduce employment. The Herfindahl index is also negatively related to the competitiveness employment growth rate. This suggests that knowledge spillovers and industry agglomeration effects may occur between industries, which is consistent with Glaeser et al.'s (1992) findings. Furthermore, average firm size is statistically unrelated to employment growth, suggesting little role between the competitiveness of industry structures and employment growth. Finally, the metropolitan share of the population is unrelated to competitiveness employment growth, which indicates a limited role of knowledge spillovers and other agglomeration effects due to having a large share of the population living in metro areas, though the significance of the population of the largest SMSA in the OLS model suggests some growth effects of urbanization. Nonetheless, there may be insufficient within state time series variation in these variables to capture their relationship to employment growth.

The relationships among competitiveness employment growth with taxes, the generosity of unemployment insurance benefits, and welfare expenditures are negative and statistically significant. This suggests that government plays a role in directly affecting business costs and in setting an overall business climate. Overall, it is somewhat surprising how statistically significant the policy variables are given the mixed results in other studies (e.g., see Bartik, 1991). ${ }^{13}$ Nonetheless, the tax coefficient in column (4) suggests that increasing state and local taxes by 10 percent (i.e., by 1.033 percent of personal income) only reduces competitiveness employment growth by 0.2 percent that year. ${ }^{14}$

Regarding the demographic variables, we find a positive association between female labor force participation rates and the competitiveness employment growth rate. Similarly, there is a positive relationship between the share of the population that is married and employment growth, which indicates a possible positive association between social stability and productivity (e.g., married men are considered more stable employees). Moreover, there is a negative relationship between the share of the population under the age of 15 and employment growth. The educational attainment variables imply that there are no strong links between employment growth and human capital. Regardless, the negative coefficient on the college education attainment variable was surprising and is not easily explained. ${ }^{15}$ Finally, in the fixed effects specification, greater rates of international 
migration are insignificant in influencing employment growth. Thus, there is some evidence supporting the claim that native out-migration offsets the supply influence of international migration on employment.

Two alternative autocorrelation-corrected models were specified to examine the sensitivity of the results to the industry composition variables. The results in column (5) omit the industry mix employment growth rates and the model in column (6) also omits the industry share variables. The industry shares may be influenced by cyclical conditions. For example, the share of construction may be procyclical, suggesting that industry shares are endogenous and may bias the reduced form model's results in columns (3) and (4). Hence, these two models can be viewed as "reduced-form" models where the "endogenous" one-digit employment shares and industry mix employment growth are omitted. In general, both columns (5) and (6) show that the other results are robust to omitting the industry composition variables. The only meaningful differences are between the models shown in columns (4) and (6). In column (6), the Herfindahl index is positive and significant, which suggests that detecting whether industry knowledge spillovers and agglomeration effects are important is somewhat sensitive to fully controlling for industry composition.

Table 3 presents results using the state employment growth rate net of the U.S. employment growth rate as the dependent variable. In this case, the dependent variable equals the competitiveness employment growth rate plus the relative state growth rate due to having a different industry mix.

In Table 3, column (1) presents the OLS estimates, column (2) adds state fixed effects, column (3) corrects the model shown in column (2) for autocorrelation, while the model shown in column (4) omits the industry share variables. The only other difference between these models and those in Table 2 is that the industry mix employment variables are omitted because the impact of industry mix on employment is now endogenous-i.e., included in the dependent variable. Although we expected similar results between the competitiveness employment growth rate and the relative employment growth rate, it is surprising how close the results actually are. Thus, the findings are robust to different measures of relative state employment growth. Furthermore, the results in Table 3 are also inconsistent with a larger high-tech sector or a greater concentration of industries (i.e., Herfindahl) positively affecting employment growth.

The only real apparent difference between the results in the two tables is in the government policy variables. Specifically, the negative influence of the tax, unemployment insurance, and welfare variables appears to be larger in the relative employment case. This could suggest that government policies have a greater influence when industry mix is endogenous. Thus, taxes appear not only to in- 
fluence the competitiveness employment growth rate, but taxes may also affect the industry composition of a state.

In both sets of employment results, the state fixed effects are jointly statistically significant at the .001 percent level. ${ }^{16}$ Certainly, there is a multitude of household amenities, firm amenities and other cross-sectional differences that are part of the state fixed effects (see Duffy, 1994). ${ }^{17}$

One empirical concern is that potential endogeneity of WAGE_COMP is influencing the results. ${ }^{18}$ For instance, high employment growth rates (e.g., labor demand growth exceeding growth in labor supply) could increase wages in the state's industries faster than their respective industry wages nationally. Clearly, this could positively bias the WAGE_COMP coefficient and possibly bias the other coefficients. ${ }^{19}$ To test for this possibility, we conducted a Hausman test (MacKinnon, 1992) on the WAGE_COMP variable in the autocorrelation-corrected specifications shown in column (4) of Table 2 and column (3) of Table $3 .^{20}$ The t-statistic for the null hypothesis that potential endogeneity of the variables is not biasing the coefficients equalled 4.71 in the competitiveness employment model and 3.45 in the relative employment model. Therefore, the null hypothesis was rejected. Likewise, we also tested for the possibility that potential endogeneity of welfare expenditures biased the results (i.e., welfare expenditures and employment growth could be negatively related). In this case, the Hausman test $\mathrm{t}$-statistic for the competitiveness employment model was 0.45 and was 0.24 in the relative employment model, and thus, the null hypothesis was not rejected. Therefore, following Greene (1993) for the case when autocorrelation is present, column (7) of Table 2 and column (5) of Table 3 present the results of a 2SLS specification where WAGE_COMP is treated as endogenous. As expected, the WAGE_COMP coefficient is more negative using 2 SLS, but the remaining coefficient estimates are basically unchanged.

\section{v. CONCLUSION}

This paper appraised a variety of potential causes for differential state employment growth rates. In particular, the relative contributions of traditional business location factors were weighed against the influence of industrial composition and knowledge and technological spillovers. The results suggest an important role for industry composition in determining employment growth and therefore support the findings in Garcia-Milà and McGuire (1993). However, we extended Garcia-Milà and McGuire's analysis by examining specific avenues for industry mix to influence employment. First, if a state possesses a mix of industries that are faring relatively well at the national level, additional growth is 
generated in other sectors through an employment multiplier. Second, a mix of high-paying industries also causes additional economic activity. However, we also found that if the state's industries pay above their national industry norms, the state's economic competitiveness is damaged, which reduces employment growth. Third, even after the influence of industry mix employment growth and wages are accounted for, industry composition still has an influence on employment, suggesting that an industry's role as a buyer and a seller of inputs is important. Particularly, a positive influence was found for construction and services, while the opposite held for transportation and public utilities and finance, insurance, and real estate, but the exact sectoral composition effects should be cautiously interpreted.

The results regarding whether knowledge and technological spillovers played a significant role were mixed. In particular, in the fixed effects model, state employment growth is not positively related to the share of total employment in high-tech manufacturing. Thus, having a greater share of employment in R\&D intensive high-tech firms may not increase state employment by generating spillovers across industries or within industries. However, the positive relation between economic diversity and growth, all else equal, suggests that such spillovers may generally exist between industries.

Finally, government policies were found to influence employment growth. Taxes and government transfer programs are negatively related to employment growth. In fact, there was evidence that the influence of government policies also affects industrial mix, but our results should be viewed as tentative. Thus, government policymakers are correct to be concerned about the role that taxes and state business climate play in affecting employment. Similarly, future research should examine if government policies also influence a state's average wage level. For example, even though high-tech firms may not necessarily spur more employment, they may increase average wages, and therefore, economic welfare.

\section{ENDNOTES}

1. Romer (1994) summarizes the endogenous growth literature.

2. Comparable disequilibrium frameworks have been used in other studies. For example, using a slightly different methodology, Dalenberg and Partridge (1995) model the impact of infrastructure on metropolitan economies using a disequilibrium approach. Likewise, in Wasylenko and McGuire's (1985) model, cross-state differences in the level of taxes and other variables are assumed to influence disequilibrium employment growth rates, along with the equilibrium level of employment. Moreover, Greenwood et al. (1991) and Treyz et al. (1993) model 
the flow of migration as a function of cross-state differences in the level of expected personal income. Besides, Newman and Sullivan's (1988) survey of regional empirical methodologies indicates that disequilibrium models have the advantage of not having to explain the entire equilibrium pattern of business location, only locational changes.

3. The chief component of the first term on the right-hand-side of Equation (6) is $\lambda E_{r l}^{*} / E_{r t-1}$. Thus, factors that influence $E^{*}$, such as the number of manufacturing workers, are scaled by the size of the state. Specifically, something proportional to $E_{r t-1}$, where, due to data availability, it is not always exactly scaled by $E_{r-1}$. That is, most of the independent variables used in the empirical analysis are expressed as ratios or percent. For example, we use the share of employment in manufacturing and the percent of the population that are college graduates, not the total number of manufacturing workers or the total number of college graduates in the state. Because the speed of adjustment term $\lambda$ is subsumed into $h$, the empirical coefficients should be interpreted as the impact on current annual employment growth and not as the ultimate long-run impact on the equilibrium level of employment $E^{*}$ in the distant future. This interpretation, of course, is consistent with our stated purpose of explaining why states grow at different growth rates during any given year.

4. The competitiveness employment for state $r$ equals

$$
\text { COMP_EMP }{ }_{r t}=\left(E_{r i}-\Sigma S_{t}^{i} E_{r t-1}^{i}\right) / E_{r t-1}
$$

where COMP_EMP $P_{r t}$ is the competitiveness growth rate for state $r$ in year $t, E_{r t}$ equals state r's total employment in period $t, S_{t}^{i}$ equals the national growth rate of industry $i$ in year $t$, and $E_{r t-1}^{i}$ equals state r's employment in industry $i$. The summation is over all two-digit, nonfarm private sector industries. Alternative models of deriving the competitiveness employment term from profit-maximizing behavior are found in Chalmers and Beckhelm (1976) and Andrikopoulos (1980). Garcia-Milà and McGuire's (1993) residual measure of relative employment growth is almost identical to the competitiveness employment term. Bartik (1991) provides additional discussion of the shift-share methodology.

5. In a similar model, Terkla and Doeringer (1991) examined a series of cross-sectional regressions of state employment growth rates that controlled for the industry mix employment growth rate and five other cost-oriented variables.

6. The industry mix employment growth rate in state $r$ is defined as INDMIX_EMP ${ }_{r t}=\left(\Sigma S_{t}^{i} E_{r t-1}^{i}-E_{U S t}\right) / E_{r t}$ where $S_{i}^{i}$ is the national growth rate of industry $i$ in year $t, E_{r-I}^{i}$ is employment in 
industry $i$ in state $r$, and $E_{U S t}$ is the total U.S. employment growth rate. The summation is over all two-digit nonfarm private sector industries.

7. The sum of the durable and nondurable shares equals manufacturing's share of employment. The high-tech share of manufacturing is an additional control.

8. Small firms may simply represent firms that are expanding. However, Davis and Haltiwanger (1992) show that there is little difference in the net job creation between small and large businesses because small businesses have a high rate of job destruction.

9. Formally, the WAGE_MIX ratio for state $r$, year $t$ is calculated as WAGE_MIX ${ }_{r t}=\left(\Sigma E M P S H_{r t}^{i}{ }^{*} W_{U S t}^{i}\right) / W_{U S t}$ where $E M P S H_{r t}^{i}$ is industry i's share of state r's nonfarm private sector employment, $W_{U S t}^{i}$ is average annual U.S. earnings in industry $i$, and $W_{U S t}$ is average U.S. nonfarm private sector annual earnings. The summation is over all nonfarm private sector industries. Formally, the WAGE_COMP ratio for state $r$, year $t$ is calculated as

$W A G E_{-} C O M P_{r t}=W_{r} /\left(\Sigma E M P S H_{r t}^{i}{ }^{*} W^{i} U S_{t}\right)$

where $W_{r t}$ is average nonfarm annual earnings in state $r$. In the empirical analysis, the possible endogeneity of the wage structure measures is examined.

10. Since the mean of the dependent variable equals zero in every time period, it is unlikely that there are annual fixed effects. However, we did not dismiss the possibility that there could be spurious time-series relationships between the independent variables and employment growth that could result in statistically significant relationships for some of the variables, when in fact no actual relationship exists. Thus, we also experimented with including annual dummies to examine whether spurious relationships contaminated our results. As expected, our results were similar when the time dummies were included.

11. Although these results suggest that the industry composition coefficients are statistically significant as a group, the particular coefficients should be cautiously interpreted because industry shares could be influenced by cyclical effects, which will be further examined below.

12. In a different specification, we changed our manufacturing variables from the share of employment in durable goods and nondurable goods with an extra high-tech share variable to simply a high-tech manufacturing share variable and a non-high-tech manufacturing share variable. Even in this case, the high-tech share was usually insignificant when state fixed effects were included.

13. It is likely that positive effects from certain government expenditures somewhat offset the negative effects of the taxes used to fund them (e.g., Helms, 1985; Dalenberg and Partridge, 1995). Nonetheless, this effect would positively 
bias the tax coefficient, implying that the tax findings are even stronger than the tax coefficient suggests.

14. In sensitivity analyses, following Blanchard and Katz (1992), the nine states with the highest shares of personal income in coal, crude oil, natural gas, and other mining activities were omitted from the specification. Basically, the results were similar to the reported results, with the exception of the tax variable. Specifically, the tax results were statistically insignificant, which implies that the negative tax effect is not robust across all states, suggesting that further research may be necessary to sort out what states are most sensitive to taxation effects.

15. In an alternative specification, we regressed the average annual earnings per worker in each state on basically the same independent variables (with the exception of WAGE_MIX and WAGE_COMP). These results suggested that a one percent greater share of the population with at least a bachelor's degree increased average annual earnings by 0.7 percent. Thus, these findings indicate that states with a higher share of college graduates are associated with higher paying jobs rather than faster employment growth.

16. For example, the F-statistic for the state fixed effects in the specification shown in column (3) of Table 2 equals 4.94 and the analogous F-statistic for the relative employment regression in column (2) of Table 3 is 4.83 .

17. In further analysis, the state fixed effects coefficients were found to be very positively related to both LOG_SMSA_POP and the 1972 per capita personal income. The positive LOG_SMSA_POP association suggests that although a state with many small metropolitan areas may not experience significantly greater economic growth, having one large metropolitan area is beneficial. The positive association between the initial per capita income and the state fixed effects supports our contention that regional economies should be modelled in a disequilibrium framework. In particular, after controlling for costs and other business location factors, there was disequilibrium migration to areas with high initial levels of income or businesses continued to relocate in response to firm amenity differentials.

18. We also considered the possibility that our error terms were spatially autocorrelated (e.g., employment shocks in Wisconsin spillover into Minnesota). We tested for this possibility by assuming that spatial autocorrelation originates in bordering states and follows an error-components specification (Kelejian and Robinson, 1993, forthcoming). We assumed that the degree of spillover into a state from a neighboring state is directly proportional to the relative size of the neighboring state's nonagricultural employment compared to the other neighboring states. Nonetheless, the null hypothesis of no spatial autocorrelation could not be rejected at any reasonable level of significance using a test developed by Kelejian and Robinson. 
19. A state's relative employment growth should not in turn have any meaningful influence on either its industry mix employment (INDMIX_EMP) components or its wage mix (WAGE_MIX) compensation component because these variables are essentially determined in the national labor market (e.g., see Blanchard and Katz 1992).

20. The instruments for the Hausman test and the two-stage least square (2SLS) estimates include all of the exogenous independent variables included in the model, relative housing costs in the state (Treyz et al. 1992), and the lags of WAGE_MIX and WAGE_COMP.

\section{REFERENCES}

Andrikopoulos, Andreas A. "A Synthesis of the Production Function and ShiftShare Model." Regional Science and Urban Economics. 1980 (10):539-560.

Barro, Robert J., and Xavier Sala-i-Martin. "Convergence Across States and Regions." Brookings Papers on Economic Activity. 1991 (1): 107-182.

Bartik, Timothy J. Who Benefits From State and Local Economic Development Policies? 1991 (Kalamazoo, MI: W.E. Upjohn Institute.)

Beeson, Patricia. "Total Factor Productivity Growth and Agglomeration Economies in Manufacturing." Journal of Regional Science. 1987 (27): 183199.

Blanchard, Olivier Jean, and Lawrence F. Katz. "Regional Evolutions." Brookings Papers on Economic Activity. 1992 (1): 1-75.

Carlino, Gerald A., and Richard Voith. "Accounting for Differences in Aggregate State Productivity." Regional Science and Urban Economics. 1992 (22): 597-617.

Chalmers, James A., and Terrance L. Beckhelm. "Shift and Share and Theory of Industrial Location." Regional Studies. 1976 (10): 15-23.

Crihfield, John B. "A Structural Empirical Analysis of Metropolitan Labor Demand." Journal of Regional Science. 1989 (29): 347-371.

Crihfield, John B., and Martin P. H. Panggabean. "Growth and Convergence in U.S. Cities." Journal of Urban Economics. 1995 (38): 138-165.

Dalenberg, Douglas, and Mark D. Partridge. "The Effects of Taxes, Expenditures, and Public Infrastructure on Metropolitan Area Employment." Journal of Regional Science. 1995 (35): 617-640.

Davis, Steve J. and John Haltiwanger. "Gross Job Creation, Gross Job Destruction, and Employment Reallocation." Quarterly Journal of Economics. 1992 (107): 819-863. 
Duffy, Neal E. "The Determinants of State Manufacturing Growth Rates: A TwoDigit-Level Analysis." Journal of Regional Science. 1994 (34): 137-162.

Freeman, Richard, and James Medoff. What Do Unions Do? 1984 (New York: Basic Books).

Frey, William H. "The New White Flight." American Demographics. 1994 (16): 40-48.

Garcia-Milà, Teresa, and Therese J. McGuire. "Industrial Mix as a Factor in the Growth and Variability of States' Economies." Regional Science and Urban Economics. 1993 (23): 731-748.

Glaeser, Edward L., Hedi D. Kallal, Joseph A. Scheinkman, and Andrei Shleifer.

"Growth in Cities," Journal of Political Economy. 1992 (100): 1126-1152.

Greene, William H. Econometric Analysis. 1993 (New York: Macmillan).

Greenwood, Michael J., Gary L. Hunt, Dan S. Rickman, and George I. Treyz.

"Migration, Regional Equilibrium, and the Estimation of Compensating Differentials." American Economic Review. 1991 (81): 1382-1390.

Helms, L. Jay. "The Effect of State and Local Taxes on Economic Growth: A Time Series Approach." Review of Economics and Statistics, 1985 (67): $574-$ 582.

Henderson, Vernon. "Where Does an Industry Locate?" Journal of Urban Economics. 1994 (35): 83-104.

Hirsch, Barry T., and David A. Macpherson. "Union Membership and Coverage Files from the Current Population Surveys: Note," Industrial and Labor Relations Review. 1993 (46): 574-578.

Hulten, Charles R., and Robert M. Schwab. "Regional Productivity Growth in U.S. Manufacturing: 1951-1978." American Economic Review. 1984 (74): 152-162.

Jorde, Thomas M., and David J. Teece. "Innovation and Cooperation: Implications for Competition and Antitrust." Journal of Economic Perspectives. 1990 (4): 75-96.

Kelejian, Harry H., and Dennis P. Robinson. "Infrastructure Productivity Estimation and its Underlying Econometric Specifications: A Sensitivity Analysis." Papers in Regional Science (forthcoming).

Kelejian, Harry H., and Dennis P. Robinson. "A Suggested Method of Estimation for Spatial Interdependent Models with Autocorrelated Errors, and an Application to a County Expenditure Model." Papers in Regional Science. No. 3, 1993 (72): 297-312.

Lucas, Robert E. "On the Mechanics of Economic Development." Journal of Monetary Economics. 1988 (22): 3-42.

MacKinnon, James G. "Model Specification Tests and Artificial Regressions." Journal of Economic Literature. 1992 (30): 102-146. 
Malhotra, Devinder M., and Gasper A. Garofalo. "Analysis of Regional Productivity with Capital as a Quasi-Fixed Factor." Regional Science and Urban Economics. 1988 (18): 533-547.

Newman, Robert J., and Dennis H. Sullivan. "Econometric Analysis of Business Tax Impacts on Industrial Location: What Do We Know, and How Do We Know It?" Journal of Urban Economics. 1988 (23): 215-234.

Partridge, Mark D., and Dan S. Rickman. "Differences in State Unemployment Rates: The Role of Labor and Product Market Structural Shifts." Southern Economic Journal. 1995 (62): 89-106.

Porter, Michael E. The Competitive Advantage of Nations. 1990 (New York: The Free Press).

Rauch, James E. "Productivity Gains from Geographic Concentration of Human Capital: Evidence from the Cities." Journal of Urban Economics. 1993 (34): 380-400.

Romer, Paul M. "The Origins of Endogenous Growth," The Journal of Economic Perspectives. 1994 (8): 3-22.

Terkla, David G., and Peter B. Doeringer. "Explaining Variations in Employment Growth: Structural and Cyclical Change among States and Local Areas," Journal of Urban Economics. 1991 (29): 329-348.

Treyz, George I., Dan S. Rickman, Gary L. Hunt, and Michael J. Greenwood. "The Dynamics of U.S. Internal Migration." Review of Economics and Statistics. 1993 (75): 209-214.

Treyz, George I., Dan S. Rickman, and Gang Shao. "The REMI EconomicDemographic Forecasting and Simulation Model." International Regional Science Review. 1992 (14): 221-253.

Trott, Edward A. Jr., Anne E. Dunbar, and Howard L. Friedenberg. "Gross State Product by Industry, 1977-89," Survey of Current Business. 1991 (71): 4359.

U.S. Bureau of the Census. Census of Population, Decennial. 1970, 1980, 1990. (Washington, D.C.: Government Printing Office).

U.S. Bureau of Economic Analysis. Local Area Personal Income. Various years. (Washington, D.C.: Government Printing Office).

U.S. Bureau of Labor Statistics. ES-202 Employment Data Series. Various years.(Washington, D.C.: Government Printing Office).

U.S. Department of Commerce. County Business Patterns. Various years. (Washington, D.C.: Government Printing Office).

. Government Finances. Various years. (Washington, D.C.: Government Printing Office).

. Statistical Abstract of the United States. Various years. (Washington,

D.C.: Government Printing Office). 
U.S. Employment and Training Administration. Unemployment Insurance Data Summary. Various years. (Washington, D.C.: Government Printing Office).

Wasylenko, Michael, and Therese McGuire. "Jobs and Taxes: The Effect of Business Climate on States' Employment Growth Rates." National Tax Journal. 1985 (38): 497-511. 